\title{
Differences between Internal Jugular Vein and Vertebral Vein Flow Examined in Real Time with the Use of Multigate Ultrasound Color Doppler
}

\author{
G. Ciuti, D. Righi, L. Forzoni, A. Fabbri, and A. Moggi Pignone
}

\begin{abstract}
BACKGROUND AND PURPOSE: The hypothesis that MS could be provoked by a derangement of the blood outflow from the brain has been largely discredited. In part, it was because data on the normal pattern of outflow are scarce and obtained with different methods. The aim of this study was to evaluate the normal pattern of outflow for the vertebral and internal jugular veins in healthy subjects with multigate color Doppler.
\end{abstract}

MATERIALS AND METHODS: Twenty-five volunteers were studied to assess vessel area, mean velocity, and flow for the vertebral and internal jugular veins in the supine and sitting positions.

RESULTS: In the sitting position, flow decreases, both in vertebral veins and internal jugular veins, as the total vessel area decreases (from $0.46 \pm 0.57$ to $0.09 \pm 0.08 \mathrm{~cm}^{2}$ ), even if the mean velocity increases (from $12.58 \pm 10.19$ to $24.14 \pm 17.60 \mathrm{~cm} / \mathrm{s}$ ). Contrary to what happens to the blood inflow, outflow in the supine position, through vertebral and internal jugular veins, is more than twice the outflow in the sitting position (739.80 \pm 326.32 versus $278.24 \pm 207.94 \mathrm{~mL} / \mathrm{min})$. In the sitting position, on application of very low pressure to the skin with the sonography probe, internal jugular veins rarely appear to occlude. A pronounced difference of diameter between internal jugular veins was present in approximately one-third of subjects.

CONCLUSIONS: Our results support the view that other outflow pathways, like the vertebral plexus, play a major role in the normal physiology of brain circulation and must be assessed to obtain a complete picture of blood outflow.

ABBREVATIONS: $\mathrm{VV}=$ vertebral vein; IJV = internal jugular vein; $\mathrm{BF}=$ blood flow; $\mathrm{QDP}=$ Quality Doppler Profiles

$\mathrm{S}$ ome authors have found that MS is associated with chronic cerebrospinal venous insufficiency syndrome, characterized by stenoses or obstructions of the internal jugular vein (IJV) and/or azygos veins, with disturbed flow and formation of collaterals. ${ }^{1,2}$

Received November 21, 2012; accepted after revision January 28, 2013.

From SOD Medicina Interna ad Orientamento all'Alta Complessità Assistenziale 3 (G.C., A.M.P., A.F.), Dipartimento di Medicina Interna e di Urgenza, and SOD Valutazione Cardiologica (D.R.), Dipartimento del Cuore e dei Vasi, Azienda Ospedaliero-Universtiaria Careggi, Firenze, Italy; and Esaote S.p.A (L.F.), Firenze, Italy.

Authorship details: G.C., concept and design of the study, analysis and interpretation of data, critical writing and revising of the intellectual content, final approval of the version to be published; D.R., concept and design of the study, analysis and interpretation of data, critical writing and revising of the intellectual content, final approval of the version to be published; L.F., concept and design of the study, analysis and interpretation of data, critical writing and revising of the intellectual content, final approval of the version to be published; A.F., analysis and interpretation of data, critical writing and revising of the intellectual content, final approval of the version to be published; A.M.P., critical writing and revising of the intellectual content, final approval of the version to be published.

Please address correspondence to Gabriele Ciuti, MD, SOD Medicina Internal Orientamento all'Alta Complessità Assistenziale 3, AOU Careggi, 50134 Firenze, Italy; e-mail: gabriele.ciuti@gmail.com

http://dx.doi.org/10.3174/ajnr.A3557
However, the blood flow in IJVs is received from the superior sagittal sinus and the straight sinus via the transverse and sigmoid sinuses as well as from the cavernous sinus via the inferior petrosal sinus. After that, the IJV flow returns in the vena cava through the brachiocephalic trunk.

The extrajugular venous system is more complex, and it can be divided into intraspinal and extraspinal compartments. Vertebral veins (VVs) are part of this system and can be considered as potential collectors of these compartments. ${ }^{3}$

According to some studies, the extrajugular venous system is fundamental for cerebral outflow drainage because it can be sufficient alone to take over the entire venous drainage of the brain. ${ }^{4-8}$

With body position change, the outflow in IJVs appears to be mostly present in the supine position, whereas it is markedly reduced during standing position, with a tendency of IJVs to collapse ${ }^{9-11}$; at the same time, a concomitant flow rise in the VVs was seen.

According to the study by Shreiber et $\mathrm{al}^{7}$, the magnitude of flow increase in the VVs did not match the decrease measured in the IJVs; 
therefore, a more complex extrajugular drainage must be considered. $^{7}$

On this basis, most authors think that chronic cerebrospinal venous insufficiency simply does not exist, ${ }^{3,12}$ that findings previously reported in some patients can be a normal variant, and that other important drainage pathways such as the VVs and the vertebral plexus ${ }^{6,7}$ must be assessed.

Moreover, outflow through IVJs and VVs is often asymmetric between the 2 sides of the neck, even in healthy subjects. ${ }^{2}$

Because physiologic data on the cerebral venous outflow are scarce and obtained by the use of different techniques, we performed a study to evaluate the normal physiology of both the VVs and IJVs in healthy subjects by use of multigate color Doppler.

\section{MATERIALS AND METHODS \\ Research Design}

The aim of this study is to investigate the physiology of IJVs and VVs in a cohort of healthy subjects with multigate echo color Doppler ultrasonography in the supine and sitting positions.

\section{Subjects and Materials}

In this study, 25 young, healthy volunteers were enrolled [ 12 men and 13 women; mean age, 27 years (range, 24-52)]. In all subjects, no IJV and/or VV lesions or previous catheter insertions in the IJV were reported, nor was a disease involving neck organs present. All patients underwent echo color Doppler ultrasonography examination of IJVs and VVs on both sides. Ultrasound examinations were performed by an expert vascular sonographer on all subjects' IJVs, and VVs were examined bilaterally from their distal part, connected to the subclavian and brachiocephalic veins, to the proximal part, represented by the presence of the mandible. Vessel area of the IJV was measured in the horizontal plane in the B-mode image at cricoid cartilage level. In the VVs, diameter measurements were obtained in the sagittal plane in the V1 segment, between the origin of $\mathrm{VV}$ and the intervertebral foramina, and the area was calculated by [(diameter of vessel/2) $2 \times \pi]$.

Venous blood volume flow (BF) $\left(\mathrm{cm}^{3} / \mathrm{min}\right)$ for IJVs and VVs was calculated as [(average velocity $\times$ area of vessel $) \times 60$ ].

Morphologic and functional characteristics of both IJVs and VVs were investigated in the supine $\left(0^{\circ}\right)$ and upright sitting $\left(90^{\circ}\right)$ positions. Image acquisitions were performed by use of a MyLab 50 Gold sonography system (Esaote S.p.A., Firenze, Italy) equipped with a linear array transducer probe (LA332, Esaote S.p.A.; operating bandwidth, 3-11 MHz; imaging frequencies, 3.5-5.0-6.6-10.0 MHz; Doppler frequencies, 3.3-5.0 MHz).

Each session started with the subject in supine position, and it then shifted to sitting position. A sufficient level of hydration of the subject during the 12 hours before the examination was recommended. A proper rigid head support was used to prevent hypo-extension or hyperextension of the neck and turning to the left or right side.

A thick layer of ultrasound gel (Aquasonic 100; Parker Laboratories, Fairfield, New Jersey) was used to ensure a complete coupling between the transducer and the examined subject's skin to avoid black cones and dark areas on the ultrasound image and to prevent excessive pressure on the examined neck to preserve the IJV shape and dimension.

\section{Quality Doppler Profiles Technology}

Quality Doppler Profiles (QDP) is an innovative multigate spectral Doppler technology that processes the echo signals backscattered from multiple depths along the ultrasound beam, producing and displaying in real-time the so-called spectral profile. ${ }^{13}$ This is a matrix of power spectral densities corresponding to the simultaneously investigated depths. ${ }^{14,15}$ The spectral profile is obtained by calculating, through the classic Fast Fourier Transform algorithm, the Doppler spectrum of 128 samples, gathered from 1 depth and by repeating the procedure over 128 or 256 consecutive depths (covering a total length of $5 \mathrm{~cm}$ ). As a result, the QDP approach extends the known benefits of spectral analysis ${ }^{16}$ to a large depth range without sacrificing the axial resolution. QDP technology investigates the "third dimension of Doppler" in a graphical form, in which spatial distribution is on the vertical axis and velocity on the horizontal one; the brightness of any pixel describes the power of the corresponding spectral attenuation. In this way, QDP enables the simultaneous analysis of different vessels (and different blood flow components within the same vessel) in real time without frame rate loss.

\section{Transducer Design}

The shape and the weight of the transducer are ergonomically important to preserve the sonographer's comfort and musculoskeletal integrity while scanning. The linear probe used for the above examinations was designed to be gripped in different ways to release the muscular effort on the wrist and on the hand during the scanning session. The longer the duration of the examination, the more useful was the possibility of changing the probe grip: the average time for the performed scanning was 40 minutes.

Furthermore, the so called "palm-hold" eased the correct orientation of the probe during transverse scanning to avoid the Doppler spectrum mirroring effect obtained when the transducer was perpendicular to the blood flow. Vein compression had to be completely avoided to preserve vein dimensions and hemodynamic characteristics: even a small external pressure can cause a huge collapse of the IJV.

This was facilitated by the low probe weight ( $80 \mathrm{~g}$ plus $170 \mathrm{~g}$ for the entire cable).

The probe used in the examinations had a tip dimension of $33 \times 8 \mathrm{~mm}$, with an externally mounted soft silicon acoustical lens without sharp angles to avoid uncomfortable contact in the scanning area for the examined subjects.

The optimized probe design allowed an easier scan; when the subject had a fat or short neck, or when venous valves assessment forced the operator to work in the neck area close to the clavicle, the narrow probe tip ensured the desired field of view. ${ }^{17}$

\section{Statistical Analysis}

For statistical analysis, we used the Student $t$ test for paired samples to compare differences of area and BF between supine and standing positions. A $P$ value of $<.05$ was considered significant.

\section{RESULTS}

Mean and standard deviation (SD) of cross-sectional area and BF of both IJVs and VVs in supine and standing positions are shown in Tables 1 and 2. The area ratio between the right and left sides, in 
Table 1: Velocity and flow analysis in internal jugular vein and vertebral vein in supine and standing positions

\begin{tabular}{lcclcc}
\hline & \multicolumn{2}{c}{ Velocity, $\mathrm{cm} / \mathrm{s}^{\mathrm{a}}$} & & \multicolumn{2}{c}{ Flow, $\mathrm{mL} / \mathrm{min}^{\mathrm{a}}$} \\
\cline { 2 - 3 } \cline { 5 - 6 } & Supine Position & Standing Position & & Supine Position & Standing Position \\
\hline IJV right & $20.58 \pm 12.6$ & $27.33 \pm 21.24$ & & $357.03 \pm 230.6$ & $108.5 \pm 117.0$ \\
IJV left & $12.71 \pm 9.23$ & $29.32 \pm 20.35$ & & $309.06 \pm 233.31$ & $126.97 \pm 135.25$ \\
Total & $16.65 \pm 11.63$ & $28.33 \pm 20.61$ & & $666.10 \pm 270.45^{\mathrm{b}}$ & $235.49 \pm 202.35^{\mathrm{b}}$ \\
VV right & $9.60 \pm 7.00$ & $19.76 \pm 12.34$ & & $41.25 \pm 150.03$ & $21.81 \pm 24.02$ \\
VV left & $7.45 \pm 5.64$ & $20.17 \pm 16.61$ & & $32.45 \pm 136.65$ & $20.94 \pm 26.45$ \\
Total & $9.60 \pm 7.01$ & $19.64 \pm 12.86$ & & $73.70 \pm 197.31$ & $42.75 \pm 38.13$ \\
IJV + VV total & $12.58 \pm 10.19$ & $24.14 \pm 17.60$ & & $739.80 \pm 326.32^{\mathrm{b}}$ & $278.24 \pm 207.94^{\mathrm{b}}$ \\
\hline a Mean \pm SD. & & & & & \\
${ }_{\text {b } P<.01 .}$ & & & & &
\end{tabular}

Table 2: Differences of area between internal jugular vein and vertebral vein in supine and standing positions

\begin{tabular}{lcc}
\hline & \multicolumn{2}{c}{ Area, $\mathbf{c m}^{2 \mathrm{a}}$} \\
\cline { 2 - 3 } & Supine Position & Standing Position \\
\hline IJV right & $0.42 \pm 0.40$ & $0.07 \pm 0.06$ \\
IJV left & $0.41 \pm 0.25$ & $0.07 \pm 0.06$ \\
Total & $0.83 \pm 0.56$ & $0.14 \pm 0.09$ \\
VV right & $0.02 \pm 0.01$ & $0.02 \pm 0.01$ \\
VV left & $0.06 \pm 0.25$ & $0.02 \pm 0.01$ \\
Total & $0.08 \pm 0.25$ & $0.03 \pm 0.02$ \\
IJV + VV total & $0.91 \pm 0.61$ & $0.17 \pm 0.09$ \\
\hline
\end{tabular}

a Mean $\pm S D$

Table 3: Side area differences in internal jugular vein and vertebral vein

\begin{tabular}{lcc}
\hline & Ratio $>2$ & Right Smaller \\
\hline IJV supine & $9 / 25(36 \%)$ & $6 / 9(67 \%)$ \\
VV supine & $15 / 25(60 \%)$ & $5 / 15(34 \%)$ \\
\hline
\end{tabular}

supine position, was $\geq 2$ in 9 subjects (36\%) for IJV and in 15 (60\%) for VV (Table 3). There was no significant side difference between the cross-sectional area and blood flow either in the supine position $(P=.9$ for IJV, $P=.8$ for $\mathrm{VV})$ or the sitting position $(P=.9$ for IJV, $P=.7$ for $\mathrm{VV})$. Instead, body position influenced both the cross-sectional area and BF: in fact, a reduction of 430.61 $(64.6 \%)$ and $30.25(41.0 \%) \mathrm{mL} / \mathrm{min}$ was present in both IJVs and VVs, respectively; however, the difference of BF was statistically significant $(P<.01)$ only for IJVs. The reduction of total BF (IJVs + VVs) between the 2 body positions was 461.56 (62.4\%) and was significantly different $(P<.01)$. BF in both IJVs and VVs was more modulated in the supine position than in the sitting position $(P<.01)$ (Fig 1$)$.

\section{DISCUSSION}

In this study, we have shown that the BF in both IJVs and VVs is significantly reduced between supine and sitting positions, confirming the data of other studies. ${ }^{11,18}$

Because the arterial BF passing from the supine to the sitting position is slightly reduced ${ }^{19}$ or increased, ${ }^{20}$ additional paths of cerebral outflow should be strongly considered.

The reduction in total venous outflow that we found in the sitting position was approximately $60 \%$, which is inferior to some studies ${ }^{11}$ but comparable to another study that used the MR imaging technique. ${ }^{18}$

We found that the area of IJVs and even VVs is different between the 2 sides of the neck, that the BF is asymmetric in many patients, and that the BF in IJVs is more modulated in sitting than supine position.

In a similar study conducted by Valdueza et $a l,{ }^{11,21}$ who examined the postural dependence of cerebral venous outflow through IJVs and VVs in 23 young healthy adults by use of colorcoded duplex sonography, measurements were taken with the body at $0^{\circ}$, $+15^{\circ},+30^{\circ},+45^{\circ}$, and $+90^{\circ}$.

The authors found that in IJVs, BF was reduced, whereas in VVs, it was increased when the subjects moved from a $0^{\circ}$ to a $90^{\circ}$ position; however, this increase was not sufficient to compensate for the drop in jugular flow, therefore, total BF was significantly reduced.

In the sitting position, half of the IJVs collapsed completely and had no flow visible (23 of 46 vessels); in 9 (39\%) subjects, the flow was absent in both IJVs.

These last data are not confirmed by our study; we think it is because of the increased accuracy and sensibility of modern machines as compared with the ones used in the previous study and in the technique that we used to avoid compression of the partially collapsed jugular veins.

Moreover, we found that BF in VVs was reduced with the body at $90^{\circ}$; we think it could be explained by the different point of $\mathrm{BF}$ sampling because we recorded VV BF in V1, between the origin of the $\mathrm{VV}$ and the intervertebral foramina, where the vein diameter is more likely to change than in the rigid V2 section. Moreover, a different regulation of the pulsed Doppler filter could in part explain these differences.

Alperin et $\mathrm{al}^{18}$ conducted a study on 10 volunteers by use of an MR imaging technique to assess effects of posture on intracranial physiology.

The study showed that a shift of the venous outflow from the IJVs to secondary venous pathways occurred in the upright posture and was also less pulsatile (57\%).

Our study confirms variations of pulsatility of BF in the $0-90^{\circ}$ body position by use of the echo color Doppler ultrasonography technique, and our BF measurements are similar to those obtained in this study.

We found considerable side differences of IJV BF, in agreement with other studies by use of different techniques, including MR imaging, PET, and echo color Doppler ultrasonography, ${ }^{22}$ and this can be explained by a frequent side dominance of venous drainage. ${ }^{23}$ Again, in accordance with the previous studies, we found a right IJV outflow dominance.

A large intersubject variability was also seen in cerebral blood outflow, as has been reported in other studies. $7,11,18,22$

The reasons for these differences are not completely understood and are probably due to many factors such as age $e^{24}$ and a wide range of global arterial inflow. ${ }^{25-27}$

The existence of different outflow pathways can also play a role because they can have a different effect in different subjects.

Echo color Doppler ultrasonography can easily be used to evaluate not only the IJV diameter differences but also the flow and flow patterns and probably can be used as a sophisticated 
A

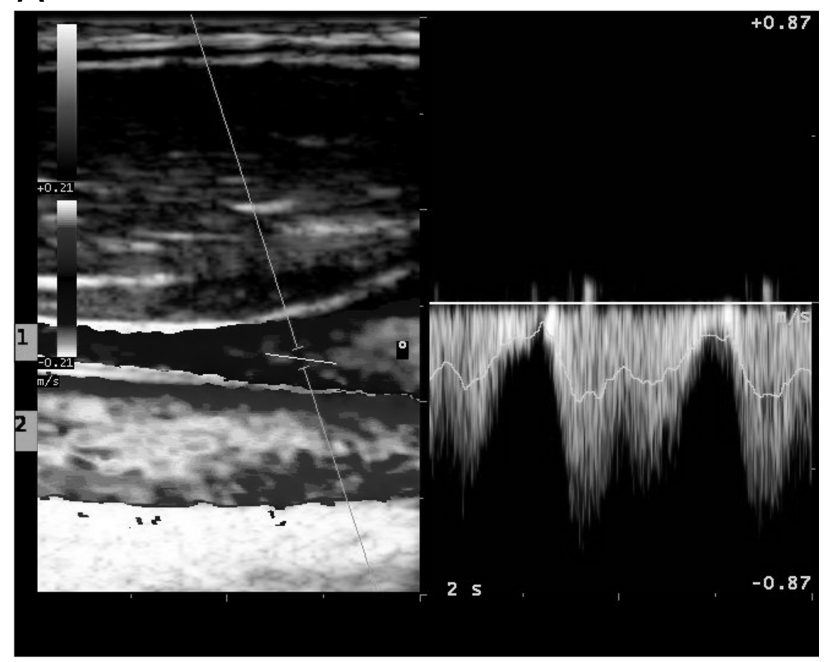

$\mathrm{B}$

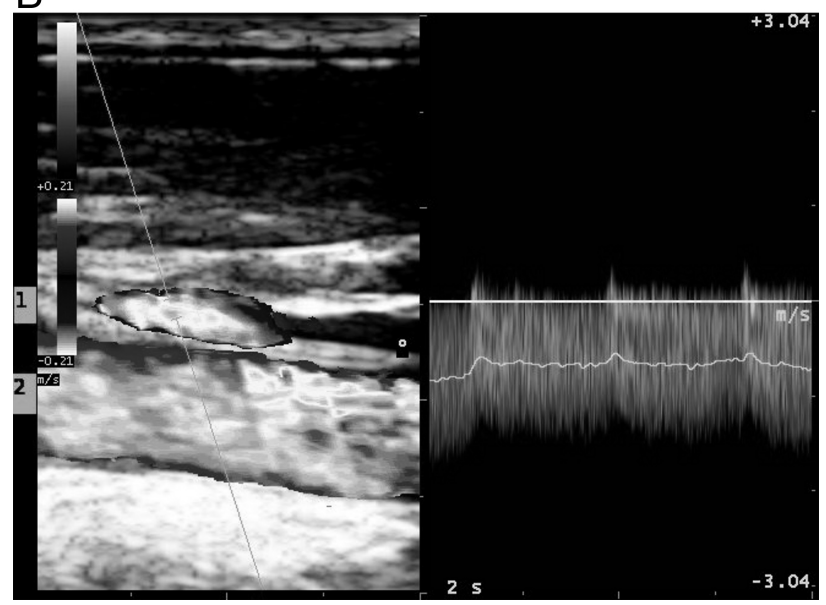

FIG 1. The pulse wave analysis shows how signal in internal jugular vein is more modulated in supine position (A) than in standing position (B). In each frame, both the internal jugular vein (1) and the external carotid artery (2) are shown.

guide to evaluate which IJV can be used for intravenous infusion devices.

In contrast to other studies, we verified that the IJV rarely occludes during the sitting position: it is not simple in this position to examine the neck, and in particular, the IJVs; it is easy to compress the IJV under the probe, so it is important to be careful and to have adequate support and an abundant quantity of ultrasonic gel to avoid the IJV collapse.

We found that QDP technology, which is able to elaborate the Doppler spectrum along all the vessel lumen, showing the spatial distribution, the velocity, and the power spectral attenuation in real time, was useful to give us a qualitative idea of how the diameter of the vessel and the velocity inside it varied with time and with the position of our subjects (Fig 2).

\section{CONCLUSIONS}

According to our data, cerebral outflow changes with body position, diminishing to less than half in the IJVs and in the VVs, and the increase in mean flow velocity is not sufficient to compensate
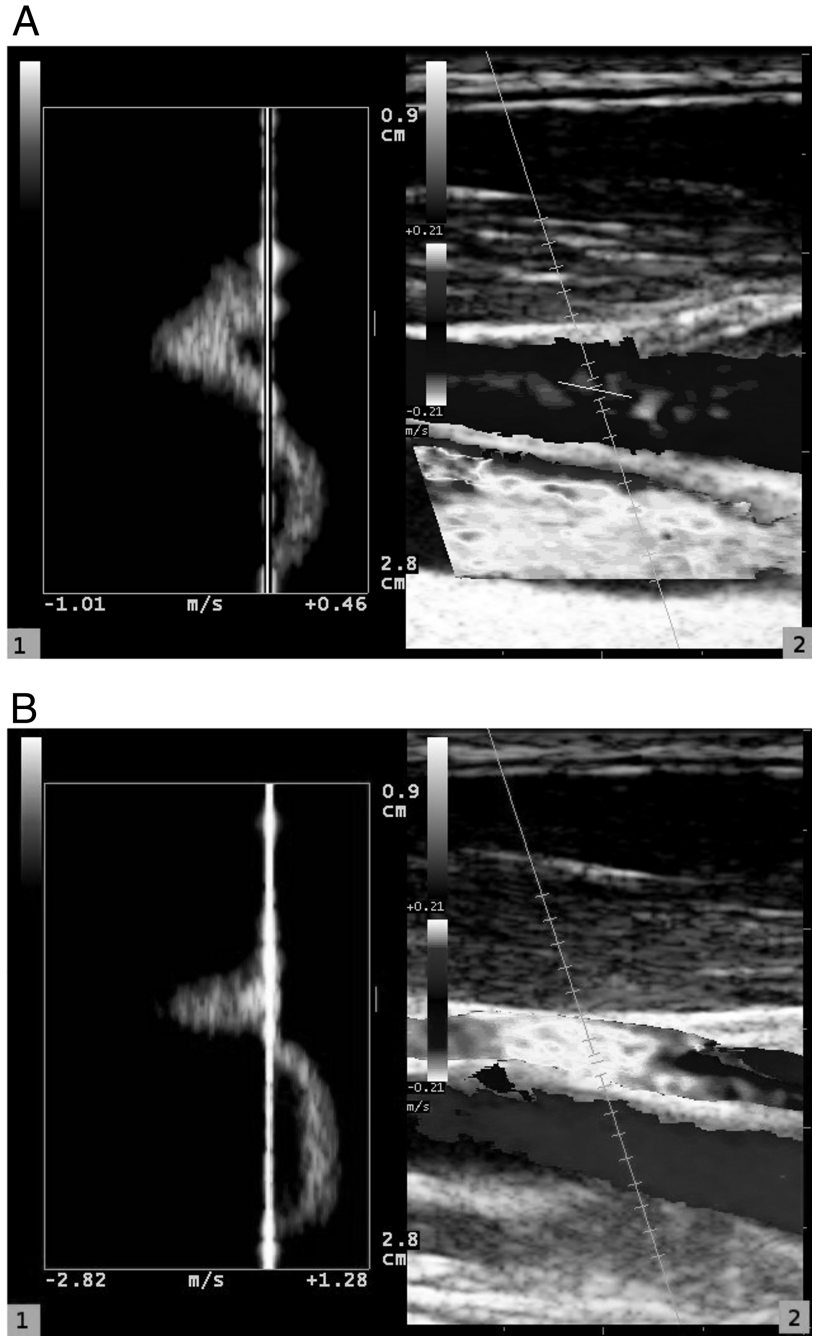

FIG 2. Blood flow in internal jugular vein analyzed by use of QDP technology (7); reference image shows internal jugular vein and common carotid artery (2). Internal jugular vein is analyzed both in supine position (A) and in standing position (B).

for the decrease of the cross-section of these vessels in the upright position.

Because arterial inflow is not so dramatically diminished, alternative outflow pathways must play a role and must be studied if any conclusion must be drawn from cerebral outflow impairment.

Because the suspected pathway is the venous plexus, which is situated inside the vertebral channel, sonography methods that are impervious to thick bone are probably inadequate to study such an anatomic structure.

The large difference that exists in a large number of subjects between the IJV area in the 2 sides of the neck makes the study of only 1 side poorly suited to draw firm conclusions and suggests that a sonography study of both veins could be useful to choose which side to use for the insertion of intravenous devices.

Disclosures: Leonardo Forzoni-UNRELATED: Employment: Esaote S.p.A., Comments: I am an employee of Esaote S.p.A., the company that implemented the QDP technology used in the study.

AJNR Am J Neuroradiol 34:2000-04 Oct 2013 www.ajnr.org 


\section{REFERENCES}

1. Zamboni P. The big idea: iron-dependent inflammation in venous disease and proposed parallels in multiple sclerosis. $J$ R Soc Med 2006;9911:589-93

2. Zamboni P, Morovic S, Menegatti E, et al. Screening for chronic cerebrospinal venous insufficiency (CCSVI) using ultrasound: recommendations for a protocol. Int Angiol 2011;306:571-97

3. Doepp F, Paul F, Valdueza JM, et al. No cerebrocervical venous congestion in patients with multiple sclerosis. Ann Neurol 2010;682: $173-83$

4. Gius JA, Grier DH. Venous adaptation following bilateral radical neck dissection with excision of the jugular veins. Surgery 1950; 282:305-21

5. Fitz-Hugh GS, Robins RB, Craddock WD. Increased intracranial pressure complicating unilateral neck dissection. Laryngoscope 1966;765:893-906

6. Batson OV. The vertebral vein system: Caldwell lecture, 1956. Am J Roentgenol Radium Ther Nucl Med 1957;782:195-212

7. Schreiber SJ, Lurtzing F, Gotze R, et al. Extrajugular pathways of human cerebral venous blood drainage assessed by duplex ultrasound. J Appl Physiol 2003;945:1802-05

8. San Millán Ruíz D, Gailloud P, Rüfenacht DA, et al. The craniocervical venous system in relation to cerebral venous drainage. $A J N R$ Am J Neuroradiol 2002;239:1500-08

9. Holt JP. The collapse factor in the measurement of venous pressure: the flow of fluid through collapsible tubes. Am J Physiol 1941; 134:292-99

10. Cirovic S, Walsh C, Fraser WD, et al. The effect of posture and positive pressure breathing on the hemodynamics of the internal jugular vein. Aviat Space Environ Med 2003;742:125-31

11. Valdueza JM, Von Münster T, Hoffman O, et al. Postural dependency of the cerebral venous outflow. Lancet 2000:3559199:200-01

12. FDA issues alert on potential dangers of unproven treatment for multiple sclerosis. http:/www.fda.gov/NewsEvents/Newsroom/ PressAnnouncements/ucm303538.htm. Accessed Jan 20, 2013

13. Tortoli P, Guidi G, Berti P, et al. An FFT-based flow profiler for high-resolution in vivo investigations. Ultrasound Med Biol 1997; 236:899-910

14. Tortoli P, Ricci S, Andreuccetti F, et al. Detection of chronic cerebrospinal venous insufficiency through multigate quality Doppler profiles. In: Ultrasonics Symposium (IUS), 2010 IEEE 2010:1190-93

15. Forzoni L, Morovic S, Semplici P, et al. Detection of reflux in jugular and vertebral veins through directional multigate quality Doppler profiles. In: Nowicki A, Litniewski J, Kujawska T, eds. Acoustical Imaging. Vol 31. Berlin, Germany: Springer-Verlag; 2012:55-67

16. Evans DH, McDicken WN. Doppler Ultrasound: Physics Instrumentation and Signal Processing, New York: Wiley; 2000

17. Forzoni L, D'Onofrio S, Farina M, et al. Combined Ultrasound Technologies and Optimized Probe Design for Neck Veins Examination. Proceedings of the IASTED Biomedical Engineering BioMed Congress 2012. Calgary, Canada: Actapress; pp. 252-59

18. Alperin N, Lee SH, Sivaramakrishnan A, et al. Quantifying the effect of posture on intracranial physiology in humans by MRI flow studies. J Magn Reson Imaging 2005;225:591-96

19. Scheinberg P, Stead EA. The cerebral blood flow in male subjects as measured by the nitrous oxide technique. Normal values for blood flow, oxygen utilization, glucose utilization, and peripheral resistance, with observations on the effect of tilting and anxiety. J Clin Invest 1949;285(Pt 2):1163-71

20. Nelson RJ, Lovick AH, Pickard JD, et al. Changes in cerebral blood flow during anaesthesia and surgery in the sitting position. J Neurol Neurosurg Psychiatry 1987;508:971-75

21. Von Münster T. [Einfluss der Körperposition auf die zerebrale venöse Drainage. Eine duplexsonographische Untersuchung der Vena jugularis interna und Vena vertebralis]. https://edoc.huberlin.de/docviews/abstract.php?id=10508. Accessed Jan 20, 2013. edoc-Server der Humboldt-Universität zu Berlin

22. Stoquart-Elsankari S, Lehmann P, Villette A, et al. A phase-contrast MRI study of physiologic cerebral venous flow. J Cereb Blood Flow Metab 2009;296:1208-15

23. Durgun B, Ilglt ET, Cizmeli MO, et al. Evaluation by angiography of the lateral dominance of the drainage of the dural venous sinuses. Surg Radiol Anat 1993;152:125-30

24. Scheel P, Ruge C, Petruch UR, et al. Color duplex measurement of cerebral blood flow volume in healthy adults. Stroke 2000;311: $147-50$

25. Scheel $P$, Ruge $C$, Schöning $M$. Flow velocity and flow volume measurements in the extracranial carotid and vertebral arteries in healthy adults: reference data and the effects of age. Ultrasound Med Biol 2000;268:1261-66

26. Schöning M, Scheel P. Color duplex measurement of cerebral blood flow volume: intra- and interobserver reproducibility and habituation to serial measurements in normal subjects. J Cereb Blood Flow Metab 1996;163:523-31

27. Schöning M, Walter J, Scheel P. Estimation of cerebral blood flow through color duplex sonography of the carotid and vertebral arteries in healthy adults. Stroke 1994;251:17-22 